\title{
Global health and its role
}

\author{
Ghulam Mustafa
}

Global health deals with the health of the populations in a worldwide context; it is defined as "the area of study, research and practice that places a priority on improving health and achieving equity in health for all people worldwide". ${ }^{1}$ A disease threat anywhere is a disease threat everywhere, and responding when and where health threats arise, disregarded to national borders, and the support is mainly from the multilateral donors. It deals with the problems which transcend the national borders or may have global political, and/or economic impact. So the global health is related with the improvement of worldwide health, which ranges from diarrheal diseases, respiratory infections including COVID-19, HIV/AIDS, and maternal health issues, among others. Such diseases have the potentials to produce disparities and global threats beyond the borders. Sometimes, global health is compared and confused with international health, which is actually a branch of public health dealing with support from the developed countries to the developing countries. Global health came outfrom the public health, and the international health, which earlier evolved from the hygiene and the tropical medicine.

The key characteristics that have a role in making the definition of the global health are; equity in the health status of the communities and their access to healthcare; a global conceptualization as against to international or between the nations perspective; causes of the health problems; means and resources for the health interventions; conceptual source of the obligation i-e its the duty of privileged to help and take care of those with fewer resources; a multidisciplinary and interdisciplinary approach means involving other professions with the health profession for improving health; stakeholders or actors such as individuals or groups; reactive or proactive approach in the provision of health globally. ${ }^{2}$

The major organizations dealing with global health are World Health Organization (WHO), UNICEF, $\mathrm{CDC}, \mathrm{PAHO}$, and Bill and Melinda Gates Foundation among others. Sustainable Development Goals and their predecessor Millennium Development Goals were formulated by United Nation to enhance comprehensive and coordinated efforts across sectors to address the socioeconomic determinants, playing role in global health threats. Global Health is measured in terms of prevalence and incidence of some the diseases, mortality from the diseases, life expectancy, DALYs, and QALYs.

The cooperation on Global Health initiatives for Global Health started with the establishment of the United Nations and the World Bank Group in 1945. Three years later in 1948, World Health Organization was formed. The concept of primary healthcare after the Alma Ata declaration in 1978 was another milestone. In the year 2000, fifteen years, eight Millennium Development Goals were formulated to address the main problems facing human development. Although these development goals were not achieved fully, nevertheless they provided the basis for accelerating a sustained human development, through 17 Sustainable Development Goals from 2015 to 2030. Among other key initiatives for Global Health included vaccine alliance GAVI in 2000, Global fund for AIDS, TB, and Malaria among others. Antimicrobial resistance, health equity, climate change, tobacco, and COVID-19 are some of the key focus areas in global health.

Some of the Global Health threats the world is facing currently are; ${ }^{3}$ Air pollution and the climate change, which results in nine out of the ten persons are globally the breathing polluted air and it is killing about 7 million people yearly prematurely from the diseases such as stroke, cancer, heart, and the lung diseases. Nearly $90 \%$ of such deaths occur in the low to the middle income countries. Non communicable diseases, which collectively cause over $70 \%$ of all the deaths worldwide making a total of about 41 million people dying yearly. This also includes about 15 million persons who are dying prematurely. Again more than $85 \%$ of such premature deaths are occurring in the low to middle-income countries. Another threat to global health is the influenza pandemic, such as COVID-19 which needs aunique partnership among all the stakeholders and players to ensure effective and equitable access or availability to the diagnostic 
services, vaccines, and antivirals (treatments), especially in low resource developing countries. One of the important and key threats to global health is fragile and the vulnerable health system, and in fact about one fourth the global population resides in crisis areas due to famine, conflict, and so the population displacement, and the weak health services delivery force them to stay without access to even the basic care. A growing issue that global health is facing is antimicrobial resistance, which has the potential to send us to state when we were not able to treat common infections like respiratory tract infections including tuberculosis, and salmonellosis. Vaccine hesitancy, which is the reluctance or refusal to get a vaccine, due to cultural, religious, and other issues. There is the availability of the vaccines, but such hesitancy of any origin threatens to halt or even reverse the progress made in controlling and eliminating the vaccine-preventable diseases globally. Another threat to global health is dengue fever, which can be a very lethal disease and it kills up to $20 \%$ of the patients with the severe form of dengue. HIV/AIDS is one of the major threats to global health and the progress made against it is enormous, but the HIV epidemic continues to kill nearly a million person yearly. Last but not the least, Global health security which is highlighted by the COVID-19 pandemic, and there is an opportunity and ground for working together to deal with the global health-related disparities and threats. The aim of the Global Health Security Agenda is to strengthen and enhance the national and international potential and ability of prevention, identification, management of the global health threats.

\section{References}

1. Jeffrey $\mathrm{P}$ Koplan, $\mathrm{T}$ Christopher Bond,Michael H Merson,K Srinath Reddy,Mario Henry Rodriguez,Nelson K Sewankambo,Judith N Wasserheit. Towards a common definition of global health. The Lancet. 2009: 373 (9679):19931995. doi:https://doi.org/10.1016/S0140-6736(09)60332-9

2. Ana Marušic'. Global health - multiple definitions, single goal. Ann Ist Super Sanità 2013:49(1);2-3

3. Global Health threats. WHO. Accessed;12th October, 2021. Available at; https://www.who.int/news-room/spotlight/tenthreats-to-global-health-in-2019

Dr. Ghulam Mustafa

Associate Professor, Department of Community Medicine, Sheikh Zayed Medical College, Rahim Yar Khan. 